\title{
Electromagnetic neutrino properties: present status and future prospects
}

\author{
Alexander Studenikin* \\ Department of Theoretical Physics, Faculty of Physics, Lomonosov Moscow State University, \\ Moscow 119991, Russia \\ Joint Institute for Nuclear Research, Dubna 141980, Moscow Region, Russia \\ E-mail: studenikesrd.sinp.msu.ru
}

There is no doubt [四]-[目] that neutrino electromagnetic properties open a window to new physics. The most general form [四] of a neutrino electromagnetic vertex function $\Lambda_{\mu}^{i j}(q)=$ $\left(\gamma_{\mu}-q_{\mu} q / q^{2}\right)\left[f_{Q}^{i j}\left(q^{2}\right)+f_{A}^{i j}\left(q^{2}\right) q^{2} \gamma_{5}\right]-i \sigma_{\mu \nu} q^{v}\left[f_{M}^{i j}\left(q^{2}\right)+i f_{E}^{i j}\left(q^{2}\right) \gamma_{5}\right]$, where $\Lambda_{\mu}(q)$ and form factors $f_{Q, A, M, E}\left(q^{2}\right)$ are $3 \times 3$ matrices in the space of massive neutrinos, in the case of coupling with a real photon $\left(q^{2}=0\right)$ provides four sets of neutrino electromagnetic characteristics: 1) the dipole magnetic moments $\left.\mu_{i j}=f_{M}^{i j}(0), 2\right)$ the dipole electric moments $\varepsilon_{i j}=f_{E}^{i j}(0)$, 3) the millicharges $q_{i j}=f_{Q}^{i j}(0)$ and 4$)$ the anapole moments $a_{i j}=f_{A}^{i j}(0)$. So far, there are no indications in favor of nonzero electromagnetic properties of neutrinos from either data from laboratory experiments with neutrino fluxes from ground-based sources or from astrophysics observations. However, the study of the electromagnetic properties of neutrinos attracts considerable attention.

The most well understood and studied are the dipole magnetic and electric moments. In a minimal extension of the Standard Model the diagonal magnetic moment of a Dirac neutrino is given [四] by $\mu_{i i}^{D}=\frac{3 e G_{F} m_{i}}{8 \sqrt{2} \pi^{2}} \approx 3.2 \times 10^{-19}\left(\frac{m_{i}}{1 \mathrm{eV}}\right) \mu_{B}$ ( $\mu_{B}$ is the Bohr magneton). Majorana neutrinos can have only transition (off-diagonal) magnetic moments $\mu_{i \neq j}^{M}$. The most stringent constraints on the effective neutrino magnetic moment are obtained with the reactor antineutrinos: $\mu_{v}<$ $2.9 \times 10^{-11} \mu_{B}$ (GEMMA Collaboration [可]), and solar neutrinos: $\mu_{v_{e}} \leq 2.8 \times 10^{-11} \mu_{B}$ (Borexino Collaboration [园]).

An astrophysical bound (for both Dirac and Majorana neutrinos) is provided [ $[$ ] $[$ [Q] by observations of the properties of globular cluster stars: $\left(\sum_{i, j}\left|\mu_{i j}\right|^{2}\right)^{1 / 2} \leq(2.2-2.6) \times 10^{-12} \mu_{B}$. A general and termed model-independent upper bound on the Dirac neutrino magnetic moment, that can be generated by an effective theory beyond a minimal extension of the Standard Model, has been derived in [ए]]: $\mu_{v} \leq 10^{-14} \mu_{B}$. The corresponding limit for transition moments of Majorana neutrinos is much weaker [ए]].

In the theoretical framework with $C P$ violation a neutrino can have nonzero electric moments $\varepsilon_{i j}$. In the laboratory neutrino scattering experiments for searching $\mu_{v}$ (for instance, in the GEMMA experiment) the electric moment $\varepsilon_{i j}$ contributions interfere with those due to $\mu_{i j}$. Thus, these kind of experiments also provide constraints on $\varepsilon_{i j}$. The astrophysical bounds on $\mu_{i j}$ are also applicable for constraining $\varepsilon_{i j}$ (see [प]-[] and [미]).

In what follows below we give a fast flash on less know neutrino electromagnetic characteristics, namely on the neutrino millicharge, charge radius and anapole moment and give some comments on the future prospects of neutrino electromagnetic properties.

The 39th International Conference on High Energy Physics (ICHEP2018)

4-11 July, 2018

Seoul, Korea

* Speaker. 


\section{Neutrino electric millicharge}

There are extensions of the Standard Model that allow for nonzero neutrino electric millicharges. This option can be provided by not excluded experimentally possibilities for hypercharhge dequantization or another new physics related with an additional $U(1)$ symmetry peculiar for extended theoretical frameworks. Neutrino millicharges are strongly constrained on the level $q_{v} \sim 10^{-21} e_{0}\left(e_{0}\right.$ is the value of an electron charge) from neutrality of the hydrogen atom.

A nonzero neutrino millicharge $q_{v}$ would contribute to the neutrino electron scattering in the terrestrial experiments. Therefore, it is possible to get bounds on $q_{v}$ in the reactor antineutrino experiments. The most stringent reactor antineutrino constraint $q_{v} \leq 1.5 \times 10^{-11} e_{0}$ is obtained in [ㄴ3] (see also [14]) with use of the GEMMA experimental data [G].

A neutrino millicharge might have specific phenomenological consequences in astrophysics because of new electromagnetic processes are opened due to a nonzero charge (see [प, प5] ). Following this line, the most stringent astrophysical constraint on neutrino millicharges $q_{v} \leq 1.3 \times 10^{-19} e_{0}$ was obtained in [ए6]. This bound follows from the impact of the neutrino star turning mechanism (STv) [ए]] that can be charged as a new physics phenomenon end up with a pulsar rotation frequency shift engendered by the motion of escaping from the star neutrinos along curved trajectories due to millicharge interaction with a constant magnetic field.

\section{Neutrino charge radius and anapole moment}

Even if a neutrino millicharge is vanishing, the electric form factor $f_{Q}^{i j}\left(q^{2}\right)$ can still contain nontrivial information about neutrino electromagnetic properties. The corresponding electromagnetic characteristics is determined by the derivative of $f_{Q}^{i j}\left(q^{2}\right)$ over $q^{2}$ at $q^{2}=0$ and is termed neutrino charge radius, $\left\langle r_{i j}^{2}\right\rangle=-\left.6 \frac{d f_{Q}^{i j}\left(q^{2}\right)}{d q^{2}}\right|_{q^{2}=0}$ (see [四] for the detailed discussions). Note that for a massless neutrino the neutrino charge radius is the only electromagnetic characteristic that can have nonzero value. In the Standard Model the neutrino charge radius and the anapole moment are not defined separately, and there is a relation between these two values: $a=-\frac{\left\langle r^{2}\right\rangle}{6}$.

A neutrino charge radius contributes to the neutrino scattering cross section on electrons and thus can be constrained by the corresponding laboratory experiments [ए]]. In all but one previous studies it was claimed that the effect of the neutrino charge radius can be included just as a shift of the vector coupling constant $g_{V}$ in the weak contribution to the cross section. However, as it has been recently demonstrated in [18]] within the direct calculations of the elastic neutrino-electron scattering cross section accounting for all possible neutrino electromagnetic characteristics and neutrino mixing, this is not the fact. The neutrino charge radius dependence of the cross section is more complicated and there are, in particular, the dependence on the interference terms of the type $g_{V}\left\langle r_{i j}^{2}\right\rangle$ and also on the neutrino mixing.

\section{Conclusions and future prospects}

The foreseen progress in constraining neutrino electromagnetic characteristics is related, first of all, with the expected new results from the GEMMA experiment measurements of the reactor antineutrino cross section on electrons at Kalinin Power Plant. The new set of data is expected 
to arrive next year. The electron energy threshold will be as low as $350 \mathrm{eV}$ ( or even lower, up to $\sim 200 \mathrm{eV}$ ). This will provide possibility to test the neutrino magnetic moment on the level of $\mu_{v} \sim 0.9 \times 10^{-12} \mu_{B}$ and also to test the millicharge on the level of $q_{v} \sim 1.8 \times 10^{-13} e_{0}$ [[ए]].

The current constraints on the flavour neutrino charge radius $\left\langle r_{e, \mu, \tau}^{2}\right\rangle \leq 10^{-32}-10^{-31} \mathrm{~cm}^{2}$ from the scattering experiments differ only by 1 to 2 orders of magnitude from the values $\left\langle r_{e, \mu, \tau}^{2}\right\rangle \leq$ $10^{-33} \mathrm{~cm}^{2}$ calculated within the minimally extended Standard Model with right-handed neutrinos [ए]]. This indicates that the minimally extended Standard Model neutrino charge radii could be experimentally tested in the near future. Note that there is a need to re-estimate experimental constraints on $\left\langle r_{e, \mu, \tau}^{2}\right\rangle$ from the scattering experiments following new derivation of the cross section [ए8] that properly accounts for the interference of the weak and charge radius electromagnetic interactions and also for the neutrino mixing. Quite recently constraints on diagonal $\left\langle r_{e, \mu, \tau}^{2}\right\rangle$ and

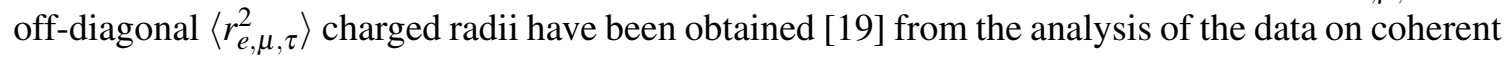
elastic neutrino-nucleus scattering obtained in the COHERENT experiment [27, 28]].

For the future progress in studying (or constraining) neutrino electromagnetic properties a rather promising claim was made in [20, 2]]. It was shown that even tine values of the Majorana neutrino transition moments would probably be tested in future high-precision experiments with the astrophysical neutrinos. In particular, observations of supernova fluxes in the JUNO experiment (see [22] - [24]) may reveal the effect of collective spin-flavour oscillations due to the Majorana neutrino transition moment $\mu_{v}^{M} \sim 10^{-21} \mu_{B}$.

\section{Acknowledgements}

This work was supported by the Russian Foundation for Basic Research under grants No. 1602-01023 A and No. 17-52-53133 GFEN_a.

\section{References}

[1] C. Giunti and A. Studenikin, Neutrino electromagnetic interactions: a window to new physics, Rev. Mod. Phys. 87 (2015) 531.

[2] A. Studenikin, Neutrino magnetic moment: a window to new physics, Nucl. Phys. Proc. Suppl. 188 (2009) 220.

[3] A. Studenikin, Neutrino electromagnetic properties: a window to new physics - II, PoS EPS-HEP2017 (2017) 137

[4] K. Fujikawa and R. Shrock, The magnetic moment of a massive neutrino and neutrino spin rotation, Phys. Rev. Lett. 45 (1980) 963.

[5] A. G. Beda, V. B. Brudanin, V. G. Egorov et al., The results of search for the neutrino magnetic moment in GEMMA experiment, Adv. High Energy Phys. 2012 (2012) 350150.

[6] M. Agostini et al. [Borexino Collaboration], Limiting neutrino magnetic moments with Borexino Phase-II solar neutrino data, arXiv:1707.09355 [hep-ex].

[7] G. G. Raffelt, New bound on neutrino dipole moments from globular-cluster stars, Phys. Rev. Lett. 64 (1990) 2856.

[8] N. Viaux, M. Catelan, P. B. Stetson, G. G. Raffelt et al., Particle-physics constraints from the globular cluster M5: neutrino dipole moments, Astron. \& Astrophys. 558 (2013) A12. 
[9] S. Arceo-Díaz, K.-P. Schröder, K. Zuber and D. Jack, Constraint on the magnetic dipole moment of neutrinos by the tip-RGB luminosity in $\omega$-Centauri, Astropart. Phys. 70 (2015) 1.

[10] N. F. Bell, V. Cirigliano, M. J. Ramsey-Musolf et al How magnetic is the Dirac neutrino?, Phys. Rev. Lett. 95 (2005) 151802.

[11] N. F. Bell, M. Gorchtein, M. J. Ramsey-Musolf, P. Vogel and P. Wang, Model independent bounds on magnetic moments of Majorana neutrinos, Phys. Lett. B 642 (2006) 377.

[12] G. G. Raffelt, Astrophysics probes of particle physics, Phys. Rept. 333 (2000) 593.

[13] A. Studenikin, New bounds on neutrino electric millicharge from limits on neutrino magnetic moment, Europhys.Lett. 107 (2014) 21001.

[14] M. Tanabashi et al. [Particle Data Group], Review of Particle Physics, Phys. Rev. D 98 (2018) no.3, 030001.

[15] G. G. Raffelt, Stars as laboratories for fundamental physics : The astrophysics of neutrinos, axions, and other weakly interacting particles, Chicago, USA: Univ. Pr. (1996) 664 p.

[16] A. Studenikin and I. Tokarev, Millicharged neutrino with anomalous magnetic moment in rotating magnetized matter, Nucl. Phys. B 884 (2014) 396.

[17] J. Bernabeu, J. Papavassiliou and D. Binosi, The neutrino charge radius in the presence offermion masses, Nucl. Phys. B 716 (2005) 352.

[18] K. Kouzakov and A. Studenikin, Electromagnetic properties of massive neutrinos in low-energy elastic neutrino-electron scattering, Phys. Rev. D 95 (2017) 055013.

[19] M. Cadeddu, C. Giunti, K. Kouzakov, Y. F. Li, A. Studenikin and Y. Y. Zhang, Neutrino charge radii from COHERENT elastic neutrino-nucleus scattering, arXiv:1810.05606 [hep-ph].

[20] A. de Gouvea and S. Shalgar, Effect of transition magnetic moments on collective supernova neutrino oscillations, JCAP 1210 (2012) 027.

[21] A. de Gouvea and S. Shalgar, Transition magnetic moments and collective neutrino oscillations: Three-flavor effects and detectability, JCAP 1304 (2013) 018.

[22] F. An et al. [JUNO Collaboration], Neutrino physics with JUNO, J. Phys. G 43 (2016) 030401.

[23] C. Giunti, K. Kouzakov, Y. F. Li, A. Lokhov, A. Studenikin, S. Zhou, Electromagnetic neutrinos in laboratory experiments and astrophysics, Annalen Phys. 528 (2016) 198

[24] J.S. Lu, Y.-F. Li and S. Zhou, Getting the most from the detection of Galactic supernova neutrinos in future large liquid-scintillator detectors, Phys. Rev. D 94 (2016) 023006.

[25] A. Grigoriev, A. Lokhov, A. Studenikin and A. Ternov, Spin light of neutrino in astrophysical environments, JCAP 1711 (2017) no.11, 024

[26] P. Kurashvili, K. A. Kouzakov, L. Chotorlishvili and A. I. Studenikin, Spin-flavor oscillations of ultrahigh-energy cosmic neutrinos in interstellar space: The role of neutrino magnetic moments, Phys. Rev. D 96 (2017) 103017

[27] D. Akimov et al. [COHERENT Collaboration], Observation of Coherent Elastic Neutrino-Nucleus Scattering, Science 357 (2017) no.6356, 1123.

[28] D. Akimov et al. [COHERENT Collaboration], COHERENT Collaboration data release from the first observation of coherent elastic neutrino-nucleus scattering, arXiv:1804.09459 [nucl-ex]. 